\title{
Colonization of long-term care facility residents in three Italian Provinces by multidrug-resistant bacteria
}

Elisabetta Nucleo ${ }^{1}$, Mariasofia Caltagirone ${ }^{1}$, Vittoria Mattioni Marchetti ${ }^{1}$, Roberto D'Angelo ${ }^{2}$, Elena Fogato ${ }^{2}$, Massimo Confalonieri ${ }^{3}$, Camilla Reboli ${ }^{3}$, Albert March ${ }^{4}$, Ferisa Sleghel ${ }^{4}$, Gertrud Soelva $^{4}$, Elisabetta Pagani ${ }^{5}$, Richard Aschbacher ${ }^{5}$, Roberta Migliavacca ${ }^{*}$, Laura Pagani ${ }^{1}$, AMCLI - GLISTer Group and ESCMID Study Group Elderly Infections - ESGIE

\begin{abstract}
Background: Rationale and aims of the study were to compare colonization frequencies with MDR bacteria isolated from LTCF residents in three different Northern Italian regions, to investigate risk factors for colonization and the genotypic characteristics of isolates. The screening included Enterobacteriaceae expressing extended-spectrum $\beta$-lactamases (ESßLs) and high-level AmpC cephalosporinases, carbapenemase-producing Enterobacteriaceae, Pseudomonas aeruginosa or Acinetobacter baumannii, methicillin-resistant Staphylococcus aureus (MRSA) and vancomycin-resistant enterococci (VRE).

Methods: Urine samples and rectal, inguinal, oropharyngeal and nasal swabs were plated on selective agar; resistance genes were sought by PCR and sequencing. Demographic and clinical data were collected.

Results: Among the LTCF residents, 75.0\% (78/104), 69.4\% (84/121) and 66.1\% (76/115) were colonized with at least one of the target organisms in LTCFs located in Milan, Piacenza and Bolzano, respectively. ES $\beta$ L producers $(60.5,66.1$ and 53.0\%) were highly predominant, mainly belonging to Escherichia coli expressing CTX-M group-1 enzymes. Carbapenemase-producing enterobacteria were found in 7.6, 0.0 and 1.6\% of residents; carbapemenase-producing $P$. aeruginosa and A. baumannii were also detected. Colonization by MRSA (24.0, 5.7 and 14.8\%) and VRE (20.2, 0.8 and 0. 8\%) was highly variable. Several risk factors for colonization by ESßL-producing Enterobacteriaceae and MRSA were found and compared among LTCFs in the three Provinces. Colonization differences among the enrolled LTCFs can be partially explained by variation in risk factors, resident populations and staff/resident ratios, applied hygiene measures and especially the local antibiotic resistance epidemiology.
\end{abstract}

Conclusions: The widespread diffusion of MDR bacteria in LTCFs within three Italian Provinces confirms that LTCFs are an important reservoir of MDR organisms in Italy and suggests that future efforts should focus on MDR screening, improved implementation of infection control strategies and antibiotic stewardship programs targeting the complex aspects of LTCFs.

Keywords: Long-term care facilities, Multicenter study, ESßL, AmpC, Carbapenemases, MRSA, VRE

\footnotetext{
* Correspondence: roberta.migliavacca@unipv.it; r.miglia@unipv.it

${ }^{1}$ Department of Clinical Surgical Diagnostic and Pediatric Sciences,

Laboratory of Microbiology and Clinical Microbiology, University of Pavia, Via

Brambilla 74, 27100 Pavia, Italy

Full list of author information is available at the end of the article
}

(c) The Author(s). 2018 Open Access This article is distributed under the terms of the Creative Commons Attribution 4.0 International License (http://creativecommons.org/licenses/by/4.0/), which permits unrestricted use, distribution, and reproduction in any medium, provided you give appropriate credit to the original author(s) and the source, provide a link to the Creative Commons license, and indicate if changes were made. The Creative Commons Public Domain Dedication waiver (http://creativecommons.org/publicdomain/zero/1.0/) applies to the data made available in this article, unless otherwise stated. 


\section{Background}

Life expectancy in Italy is rapidly increasing, with present values of 80.1 years for males and 84.7 for females [1]. Due to the ageing population, long-term care facilities (LTCFs), which provide ongoing skilled nursing care to residents and help meet both the medical and non-medical needs of elderly individuals with a chronic illness or disability, play an important role in the Italian healthcare system. Residents in LTCFs have a variety of risk factors for colonization with multidrug-resistant (MDR) bacteria; therefore, these facilities represent reservoirs of: i) Enterobacteriaceae expressing extended-spectrum $\beta$-lactamases (ES $\beta L s)$, derepressed/acquired high-level AmpC cephalosporinases or carbapenemases, ii) Pseudomonas aeruginosa or Acinetobacter baumannii producing carbapenemases and iii) methicillin-resistant Staphylococcus aureus (MRSA) and vancomycin-resistant enterococci (VRE) [2-4].

To promote detailed studies of various microbiological aspects related to LTCFs in Italy, the Association of Italian Clinical Microbiologists (Associazione Microbiologi Clinici Italiani; AMCLI) in 2016 has set up a new working group consisting of Clinical Microbiologists (Gruppo di Lavoro per lo Studio delle Infezioni nelle Residenze Sanitarie Assistite e Strutture assimilabili; GLISTer); one of the main objectives of this working group is the study of the distribution and prevalence of MDR organisms in Italian LTCFs and therefore a multicenter point-prevalence survey, including the main MDR bacteria as described above, was performed in 2016 on residents of LTCFs, located in three Northern Italian cities.

\section{Methods}

\section{The aim}

Rationale and aims of the study were to compare colonization frequencies with MDR bacteria of LTCF residents in three different Northern Italian cities, located in different Italian regions, and to investigate their genotypic characteristics. Moreover, risk factors for colonization were compared between LTCFs and colonization prevalence was correlated with the local epidemiology of invasive MDR isolates.

\section{Facilities, patient characteristics and survey design}

In October-November 2016, a multicenter pointprevalence screening study was conducted in four LTCFs concerning i) Enterobacteriaceae with ES $\beta L s$, carbapenemases or high-level AmpCs, ii) P. aeruginosa or A. baumannii with carbapenemases, iii) MRSA and VRE. The four facilities, located in the Northern Italian Provinces of Milan $(n=1)$, Piacenza $(n=2)$ and Bolzano $(n=1)$, offer high skilled $24 \mathrm{~h}$ nursing care.

Although the overall study was performed over a period of 2 months, the sampling interval in each facility lasted for a maximum of 1 week. All residents of the four LTCFs were eligible to participate, and the study was approved by the Ethics Committees of the three referring hospitals; informed written consent was obtained from the residents or, if they were unable to consent, from their relatives.

\section{Microbiological methods}

Sample processing, microbial identification and antibiotic susceptibility testing were carried out in the clinical microbiology laboratories of the referral hospitals. Microbiological methods for the LTCF screening study in Bolzano were previously described [5]. Similar methods were used in the epidemiological studies of Milan and Piacenza LTCFs, with minor modifications.

For the screening of MDR bacteria from LTCF residents in Milan midstream or catheter urine samples were cultured on Oxoid Brilliance ${ }^{\mathrm{Tm}}$ ES $\beta \mathrm{L}$ plates (Thermo Scientific, UK), applying a $10 \mu \mathrm{g}$ imipenem (IMP) disc (Oxoid, Thermo Scientific, UK), and on Oxoid Brilliance ${ }^{\text {Ts }}$ VRE (Thermo Scientific, UK). Inguinal, oropharyngeal and rectal swabs were seeded on Oxoid Brilliance ${ }^{\mathrm{Tm}}$ ES $\beta \mathrm{L}$, applying a $10 \mu \mathrm{g}$ IMP disc, on Oxoid Brilliance ${ }^{\mathrm{Tm}}$ VRE and on CHROMagar ${ }^{\mathrm{TM}}$ MRSA (BD Diagnostics, MD). Nasal swabs were plated on $\mathrm{CHROMagar}^{\mathrm{TM}}$ MRSA. All plates were incubated at $35 \pm 2^{\circ}$ $\mathrm{C}$ under aerobic conditions for 24-48 h. Isolate identification and antibiotic susceptibility testing were performed by the BD Phoenix ${ }^{\text {TM }}$ System (BD Diagnostics, MD), according to European Committee on Antimicrobial Susceptibility Testing (EUCAST) criteria [6], using PHOENIX NMIC/ ID402 for non-urinary Gram-negative bacteria, PHOENIX UNMIC/ID403 for Gram-negative isolates from urine cultures, and PHOENIX PMIC/ID88 for MRSA and VRE. The strains were phenotypically confirmed for $\beta$-lactamase production by the ESBL+AMPC Screen Kit and the KPC+ MBL Confirm ID Kit (Rosco Diagnostica A/S, Denmark).

Similarly, for screening of MDR bacteria from LTCF residents in Piacenza, midstream or catheter urine samples were seeded on ChromID CPS agar (BioMèrieux, Marcy l'Etoile, France); rectal swabs on ChromID ESBL Agar (BioMèrieux, Marcy l'Etoile, France), on ChromID VRE Agar (BioMèrieux, Marcy l'Etoile, France) and on Mac Conkey agar applying a $10 \mu \mathrm{g}$ meropenem (MER) disc (Oxoid, Thermo Scientific, UK); nasal swabs on Chapman Agar (Oxoid, Thermo Scientific, UK), on ChromID ESBL and on MacConkey agar applying a $10 \mu \mathrm{g}$ MER disc; and inguinal swabs on Mannite salt agar (Oxoid, Thermo Scientific, UK). Plates were incubated at $35 \pm 2{ }^{\circ} \mathrm{C}$ under aerobic conditions for 24-48 h. Isolate identification and antibiotic susceptibility testing were performed using the Vitek 2 System (BioMèrieux, Marcy l'Etoile, France), calibrated against EUCAST criteria [6], with AST-N202 cards (including an ESßL test) for Gram-negative bacteria, AST-P632 cards (with both oxacillin and cefoxitin) for MRSA and AST-P586 cards 
for VRE. Identification of $\beta$-lactamase types was based on Vitek 2 results and on the synergistic effects obtained by the ESBL+AMPC Screen Kit and the KPC+MBL Confirm ID Kit (Rosco Diagnostica A/S, Denmark). VRE were confirmed by vancomycin and teicoplanin Etest strips (BioMèrieux, Marcy l'Etoile, France).

\section{Molecular characterization of resistance genes}

Molecular characterization of all MDR isolates was performed in a common reference laboratory, located at the University of Pavia. Total DNA was extracted by the automated Puro extraction system (DID, Milan, Italy), using the DNA tissue kit, according to manufacturer's instructions. The presence of ES $\beta \mathrm{L}$ and carbapenemase genes was investigated by PCR, targeting $b l a_{\mathrm{CTX}-\mathrm{M}^{-}}$, bla $\mathrm{SHV}^{-} b l a_{\mathrm{KPC}^{-}}, b l a_{\mathrm{VIM}^{-}}, b l a_{\mathrm{IMP}^{-}}, b l a_{\mathrm{OXA}^{-} 48^{-}}, b l a_{\mathrm{NDM}^{-}}$ and $b l a_{\mathrm{GES}}$-type genes, and using published primers and conditions [7-15], summarized in Additional file 1: Table S1. A. baumannii isolates were screened for the presence of the following carbapenemase genes: $b l a_{\text {OXA- }}$ ${ }_{23}$-like, $b l a_{\text {OXA-24-like, }} b l a_{\text {OXA-51-like and }} b l a_{\text {OXA-58-like }}$ [16-18]. The presence of ISAba1 elements adjacent to bla $a_{\text {OXA-51-like genes was determined as previously }}$ described [19]; AmpC genes were detected by a multiplex PCR [20].

Bacterial isolates collected from the LTCF in Milan were screened for $b l a_{\mathrm{KPC}^{-}}, b l a_{\mathrm{VIM}^{-}}, b l a_{\mathrm{OXA}-48^{-}}$and $b l a_{\mathrm{NDM}^{-}}$-type genes by the Cepheid GeneXpert System and confirmed by PCR. Check-MDR CT103 XL array (Check points Health B.V., Wageningen, The Netherlands) has been used to investigate the bla gene content of a carbapenem-resistant $P$. aeruginosa strain obtained from an oropharyngeal swab, which tested negative by previous molecular assays.

For gene sequencing, PCR products were purified using the quantum Wizard ${ }^{\circ} \mathrm{SV}$ Gel and PCR Clean-Up System (Promega, Madison, WT, USA) and subjected to double-strand Sanger sequencing. Sequences were analyzed according to the BLAST software [21].

\section{Statistical analysis}

A significance level of $p \leq 0.05$ was used. In-house physicians reviewed hospital records and, using a standard questionnaire, recorded demographic and clinical data as follows: patient age, gender, length of stay, Barthel immobility score, coma, comorbidities (dementia, urinary incontinence, diabetes, cancer, vascular diseases, chronic obstructive pulmonary disease, decubitus ulcer), presence of infection, antibiotic treatment in the preceding 3 months and the presence of indwelling medical devices. The significance of differences in risk factors and colonization proportions was calculated using the proportion comparison test. Logistic regression analyses were developed to investigate colonization of at least one site with ES $\beta \mathrm{L}$ producers and MRSA as dependent variables, first as univariate and then as multivariate models, including predictors with $p<0.05$ in the univariate analysis, comprising the specific LTCF of residence, using stepwise logistic selection. Analysis were performed using the Medcalc ${ }^{\oplus}$ software version 15.11.4 (MedCalc software, Ostend, Belgium).

\section{Results}

A variable percentage of LTCF residents, present during the point-prevalence survey in the four LTCFs, agreed to participate: 104/310 (34\%) in Milan, 121/326 (37\%) in Piacenza (2 LTCFs, with 71/216 and 50/110 participating residents, respectively), and all 115 (100\%) residents in the LTCF in Bolzano; no specific LTCF resident selection criteria were used in Milan and Piacenza and resident characteristics of enrolled and not-enrolled residents were similar. The median age of LTCF residents in Milan, Piacenza and Bolzano was 82 years (range: 65-96 years), 86 years (range: 63102 years) and 77 years (range: $30-94$ years) for males, and 90 years (range: $71-102$ years), 88 years (69-105 years) and 84 years (24-96 years) for females, respectively. The median length of stay of residents in the LTCFs in Milan, Piacenza and Bolzano was 23 months (range: 1199 months), 34 months (range: 1-172 months) and 19 months (range: 1-174 months), respectively. Various healthcare staff/resident ratios were found in the LTCFs in Milan (ratio: 0.62; 193/310), Piacenza (ratio: 0.61; 201/326; corresponding to $73 / 110$ and $128 / 216$ in the two enrolled LTCFs, respectively) and Bolzano (ratio: 0.79; 91/115). Demographic and clinical details of the enrolled LTCF residents are summarized in Table 1.

Isolation frequencies and molecular characterization of the antibiotic resistance determinants are shown in Table 2. A high percentage of LTCF residents were colonized with at least one of the target MDR organisms in Milan (75.0\%; 78/104), Piacenza $(69.4 \% ; 84 / 121)$ and Bolzano (66.1\%; 76/115); moreover, many residents from Milan (37.5\%; 39/104), Piacenza (19.8\%; 24/121) and Bolzano $(30.4 \%$; $35 / 115)$ were colonized with more than one MDR organism.

ESßL-producing E. coli expressing $b l a_{\mathrm{CTX}-\mathrm{M}^{-l i k e}}$ genes were highly predominant in Milan (80.4\%), Piacenza (97.0\%) and Bolzano (80.3\%) and CTX-Mtype determinants were also identified in Proteus mirabilis, Klebsiella pneumoniae, Citrobacter koseri, Enterobacter cloacae complex and Serratia marcescens. Most $b l a_{\text {СТХ-M- }}$ genes belonged to group-1 (72.4\%), followed by group-9 (14.8\%) and other groups (12.8\%). A bla $a_{\mathrm{BEL}}$-like gene was detected in a $P$. aeruginosa strain from the LTCF in Milan.

In total, ten carbapenemase-producing Enterobacteriaceae were detected: $n=7 \mathrm{KPC}$-producing $K$. pneumoniae and $n=1$ VIM-1-producing E. cloacae 
Table 1 Demographic and clinical details of LTCF residents from three Italian Provinces

\begin{tabular}{|c|c|c|c|c|}
\hline & $\begin{array}{l}\text { Milan (M), \% } \\
(n=104)\end{array}$ & $\begin{array}{l}\text { Piacenza (P), \% } \\
(n=121)\end{array}$ & $\begin{array}{l}\text { Bolzano (B), \% } \\
(n=115)\end{array}$ & Significant differences ( $p$-value) \\
\hline Male sex & 30.7 & 26.4 & 43.4 & M vs. B (0.05); P vs. B (0.006) \\
\hline Age $\geq 86$ years & 58.7 & 60.3 & 35.6 & M vs. $B(<0.001) ; P$ vs. $B(<0.001)$ \\
\hline Antibiotics in preceding 3 months & 24.0 & 50.4 & 23.4 & M vs. $P(<0.001) ; P$ vs. $B(<0.001)$ \\
\hline Fluoroquinolones & 8.6 & 7.4 & 5.2 & \\
\hline Penicillins & 2.8 & 1.6 & 12.1 & M vs. B (0.01); P vs. B (0.001) \\
\hline Cephalosporins & 5.7 & 24.8 & 1.7 & M vs. $P(<0.001) ; P$ vs. $B(<0.001)$ \\
\hline Dementia & 42.3 & 79.3 & 68.7 & M vs. $P(<0.001) ; M$ vs. $B(<0.001)$ \\
\hline Peripheral vascular disease & 59.6 & 47.1 & 71.3 & P vs. $B(<0.001)$ \\
\hline Urinary incontinence & 74.0 & 84.3 & 85.2 & M vs. $P(0.05) ;$ M vs. $B(0.04)$ \\
\hline Diabetes & 19.2 & 16.5 & 20.8 & \\
\hline Cancer & 8.6 & 8.2 & 9.5 & \\
\hline Decubitus ulcer & 6.7 & 5.7 & 11.3 & \\
\hline Chronic obstructive pulmonary disease & 11.5 & 9.1 & 18.2 & P vs. B (0.04) \\
\hline Physical disability (Barthel immobility score of 0 ) & 10.4 & 41.3 & 67.8 & M vs. $P(<0.001) ; M$ vs. $B(<0.001) ; P$ vs. $B(<0.001)$ \\
\hline Coma & 0.0 & 0.0 & 17.4 & M vs. $B(<0.001) ; P$ vs. $B(<0.001)$ \\
\hline Any medical device & 10.5 & 23.9 & 38.2 & M vs. $P(0.009) ; M$ vs. $B(<0.001) ; P$ vs. B (0.01) \\
\hline Percutaneous enteral gastrostomy tube & 2.8 & 11.5 & 20.8 & M vs. $P(0.01) ; M$ vs. $B(<0.001) ; P$ vs. $B(0.05)$ \\
\hline Tracheostomy tube & 0.0 & 1.6 & 9.5 & M vs. B (0.001); P vs. B (0.007) \\
\hline Urinary catheter & 8.6 & 6.6 & 18.2 & M vs. B (0.04); P vs. B (0.006) \\
\hline Nasogastric tube & 0.0 & 9.1 & 1.7 & M vs. P (0.001); P vs. B (0.01) \\
\hline Length of stay in LTCF $<6$ months & 17.7 & 8.2 & 17.3 & M vs. P (0.03); P vs. B (0.03) \\
\hline \multirow{2}{*}{$\begin{array}{l}\text { Hospital admission in previous } 12 \text { months, } \\
\text { any department }\end{array}$} & \multirow[t]{2}{*}{22.3} & \multirow[t]{2}{*}{15.8} & \multirow[t]{2}{*}{38.2} & M vs. $B(0.01) ; M$ vs. $P(<0.001)$ \\
\hline & & & & P vs. $B(<0.001)$ \\
\hline Geriatrics & 0.0 & 1.6 & 9.5 & M vs. $B(p=0.001) ;$ P vs. $B(p=0.007)$ \\
\hline Medicine & 4.8 & 5.7 & 6.0 & \\
\hline Orthopedics & 3.8 & 3.3 & 4.3 & \\
\hline Infection & $3.8^{\mathrm{a}}$ & $5.7^{b}$ & $0.8^{c}$ & P vs. B (0.03) \\
\hline
\end{tabular}

a Urinary tract infection - UTI (2), respiratory tract infection - RTI (1), infected prosthesis (1)

${ }^{\mathrm{b}} \mathrm{RTI}(6)$, UTI (1), skin and soft tissue infection (1)

'UTI (1)

complex were isolated from LTCF residents in Milan, and $n=2$ VIM-1 producers (one E. coli and one Citrobacter amalonaticus) from residents in Bolzano. Two carbapenemase-positive $P$. aeruginosa were isolated from LTCF residents in Piacenza: in one case a $b l a_{\mathrm{GES}-5}$ and in the other a bla $a_{\mathrm{VIM}}$-like gene were identified. Moreover, two P. aeruginosa isolates collected in Milan and Piacenza presented a $b l a_{\mathrm{GES}-1}$ ES $\beta$ L. Nine bla $a_{\mathrm{OXA}-23}$-positive $A$. baumannii were isolated from two and seven LTCF residents in Milan and Piacenza, respectively.

MRSA strains were most frequently isolated from LTCF residents in Milan and Bolzano, whereas VRE isolates were highly prevalent in Milan ( $n=21$ Enterococcus faecalis), but rare in Piacenza $(n=1$ E. faecalis) and Bolzano ( $n=1$ Enterococcus faecium).
Colonization of LTCF residents with ES $\beta$ L-producing enterobacteria and MRSA was associated with several risk factors in univariate and multivariate analysis (Table 3). In multivariate analysis, the LTCF of residence was an independent risk factor for ES $\beta L$ ( $p \leq 0.03$ for all comparisons, except $p=0.53$ for the comparison of Milan vs. Piacenza) and MRSA ( $p \leq 0.02$ for all comparisons) colonization. Risk factors for MRSA colonization were also associated with resident's gender; for the following risk factors significant differences between male $(n=226)$ and female $(n=114)$ residents were found: age $>85$ years (M: 34.5\%; F: 20.4\%; $p<0.001$ ), hospitalization within the previous 12 months (M: $35.0 \%$; F: $20.4 \% ; p=0.03)$, administration of any antibiotic within the previous 3 months (M: 40.3\%; F: 29.6\%; $p=0.04$ ) and coma (M: $10.5 \%$ F: $3.5 \% ; p=0.009)$. 
Table 2 Colonization percentages in residents from LTCFs of three Italian Provinces

\begin{tabular}{|c|c|c|c|c|}
\hline & \multicolumn{4}{|c|}{$\begin{array}{l}\% \text { of LTCF residents colonized with specific resistance } \\
\text { phenotype and genotype and significant differences }(p \leq 0.05)\end{array}$} \\
\hline & $\begin{array}{l}\text { Milan } \\
(n=104)\end{array}$ & $\begin{array}{l}\text { Piacenza } \\
(n=121)\end{array}$ & $\begin{array}{l}\text { Bolzano } \\
(n=115)\end{array}$ & $\begin{array}{l}\text { Significant differences } \\
(p \leq 0.05)\end{array}$ \\
\hline $\begin{array}{l}\text { All resistance groups (MRSA; VRE; ESßL-/AmpC-producing enterobacteria; } \\
\text { carbapenemase-producing enterobacteria, Pseudomonas aeruginosa and } \\
\text { Acinetobacter baumannii) }\end{array}$ & 75.0 & 69.4 & 66.1 & \\
\hline All ES $\beta$ L-positive enterobacteria & 60.5 & 66.1 & 53.0 & P vs. B (0.04) \\
\hline Escherichia coli, ESßL-positive & 48.0 & 55.3 & 45.2 & \\
\hline blaCTX-M-group-1 & 33.6 & 41.3 & 28.7 & P vs. B (0.04) \\
\hline blaCTX-M-group-9 & 6.7 & 5.7 & 9.5 & \\
\hline blaCTX-M-group, other than 1 or 9 & 4.8 & 9.9 & 0.0 & P vs. $B(<0.001)$ \\
\hline Proteus mirabilis, ES $\beta$ L-positive & 14.4 & 9.1 & 7.0 & \\
\hline blaCTX-M-group-1 & 3.8 & 4.1 & 0.0 & M vs. B (0.04); P vs. B (0.03) \\
\hline blaCTX-M-group-9 & 1.9 & 0.0 & 0.0 & \\
\hline Klebsiella pneumoniae, ESBL-positive & 6.7 & 5.7 & 6.1 & \\
\hline blaCTX-M-group-1 & 5.7 & 4.1 & 1.7 & \\
\hline blaCTX-M-group-9 & 0.9 & 0.8 & 0.0 & \\
\hline blaCTX-M-group, other than 1 or 9 & 0.0 & 0.0 & 2.7 & \\
\hline Morganella morganii, ES $\beta$ L-positive & 1.9 & 1.6 & 2.6 & \\
\hline Citrobacter koseri, ESßL-positive & 0.0 & 3.3 & 0.8 & \\
\hline blaCTX-M-group other than 1 or 9 & 0.0 & 3.3 & 0.0 & \\
\hline Enterobacter cloacae complex, ES $\beta$ L-positive & 0.9 & 0.8 & 0.0 & \\
\hline blaCTX-M-group-1 & 0.0 & 0.8 & 0.0 & \\
\hline blaCTX-M-group other than 1 or 9 & 0.9 & 0.0 & 0.0 & \\
\hline Serratia marcescens, ESßL-positive & 0.0 & 0.8 & 0.0 & \\
\hline blaCTX-M-group-1, blaCTX-M-15-like & 0.0 & 0.8 & 0.0 & \\
\hline Providencia stuartii & 1.9 & 0.0 & 0.0 & \\
\hline All high-level AmpC-positive enterobacteria & 5.7 & 3.3 & 25.2 & M vs. $B(<0.001) ; P$ vs. $B(<0.001)$ \\
\hline Enterobacter cloacae complex, high-level AmpC & 0.0 & 0.8 & 0.0 & \\
\hline Morganella morganii, high-level AmpC & 3.8 & 0.8 & 24.3 & M vs. $B(<0.001) ; P$ vs. $B(<0.001)$ \\
\hline blaDHA-type & 3.8 & 0.8 & 8.7 & P vs. B (0.004) \\
\hline Citrobacter freundii, high-level AmpC & 0.0 & 0.8 & 0.0 & \\
\hline Proteus mirabilis, high-level AmpC & 1.9 & 0.0 & 0.8 & \\
\hline blaCMY-type & 0.0 & 0.0 & 0.8 & \\
\hline Serratia marcescens, high-level AmpC & 0.0 & 0.8 & 0.0 & \\
\hline Providencia rustigianii, high-level AmpC & 0.9 & 0.0 & 0.0 & \\
\hline All carbapenemase-positive enterobacteria & 7.6 & 0.0 & 1.6 & M vs. P (0.002); M vs. B (0.03) \\
\hline Klebsiella pneumoniae, blaKPC-type & 6.7 & 0.0 & 0.0 & M vs. P (0.004); M vs. B (0.05) \\
\hline Escherichia coli, blavIM-1 & 0.0 & 0.0 & 0.8 & \\
\hline Enterobacter cloacae complex, blavIM-1 & 0.9 & 0.0 & 0.0 & \\
\hline Citrobacter amalonaticus, blaVIM-1 & 0.0 & 0.0 & 0.8 & \\
\hline Carbapenemase-positive Pseudomonas aeruginosa & 0.0 & 1.6 & 0.0 & \\
\hline blaVIM-type & 0.0 & 0.8 & 0.0 & \\
\hline blaGES-5 & 0.0 & 0.8 & 0.0 & \\
\hline Carbapenemase-positive Acinetobacter baumannii & 1.9 & 5.8 & 0.0 & P vs. B (0.009) \\
\hline blaOXA-23-like & 1.9 & 5.8 & 0.0 & P vs. B (0.009) \\
\hline
\end{tabular}


Table 2 Colonization percentages in residents from LTCFs of three Italian Provinces (Continued)

\begin{tabular}{|c|c|c|c|c|}
\hline & \multicolumn{4}{|c|}{$\begin{array}{l}\% \text { of LTCF residents colonized with specific resistance } \\
\text { phenotype and genotype and significant differences }(p \leq 0.05)\end{array}$} \\
\hline & $\begin{array}{l}\text { Milan } \\
(n=104)\end{array}$ & $\begin{array}{l}\text { Piacenza } \\
(n=121)\end{array}$ & $\begin{array}{l}\text { Bolzano } \\
(n=115)\end{array}$ & $\begin{array}{l}\text { Significant differences } \\
(p \leq 0.05)\end{array}$ \\
\hline MRSA & 24.0 & 5.7 & 14.8 & M vs. $P(<0.001) ; P$ vs. $B(0.02)$ \\
\hline VRE & $20.2^{\mathrm{a}}$ & $0.8^{\mathrm{a}}$ & $0.8^{\mathrm{b}}$ & M vs. $P(<0.001) ; M$ vs. $B(<0.001)$ \\
\hline
\end{tabular}

Notes: ${ }^{\mathrm{a}}$ Enterococcus faecalis; ${ }^{\mathrm{b}}$ Enterococcus faecium

\section{Discussion}

The study evaluated the degree of colonization with drugresistant bacteria among residents of LTCFs located in three Northern Italian Provinces, finding high colonization of residents in Milan (75.0\%), Piacenza (69.4\%) and Bolzano (66.1\%). Many residents had more than one target organism, underscoring the role of LTCFs as a reservoir for these isolates [2-4].

Colonization of LTCF residents with ES $\beta$ L-producing enterobacteria was highly prevalent in all the surveyed LTCFs $(60.5 \%$ in Milan, $66.1 \%$ in Piacenza and $53.0 \%$ in Bolzano), and group-1 CTX-M-type enzymes were highly predominant, especially in E. coli (80-97\% of isolates). Notably, about $82 \%$ of K. pneumoniae and $32 \%$ of P. mirabilis isolates also harbored a $b l a_{\mathrm{CTX}-\mathrm{M}^{-t y p e}}$ gene. In the same Bolzano LTCF, here screened for ESBL-producing enterobacteria, high colonization percentages, equal to 64.0 and $49.0 \%$, were previously found in 2008 [22] and 2012 [23], respectively; the latter survey also screened a second LTCF in the Province of Bolzano, showing a colonization prevalence of $56.0 \%$. In an Italian study carried out in 2006, a colonization prevalence of $54.0 \%$ was found in LTCF residents bearing a urinary catheter [24], while a more recent multicenter study, performed in 2015 and involving 12 Italian LTCFs, reported a mean ES $\beta \mathrm{L}$ colonization of 57.3\% (range: $32.8-81.5 \%$ ) [25]. In all these Italian studies, CTX-M enzymes were the predominantly produced ES $\beta$ Ls. The high ES $\beta$ L colonization rates of > $50 \%$ in Italian LTCF residents are paralleled by high ES $\beta \mathrm{L}$ prevalence in invasive E. coli isolates [26]. Generally, ES $\beta$ L carriage in most European countries is strikingly lower than that found in Italy [4], with exceptions reported from Ireland [27, 28] and Portugal [29].

In our screening study, high-level AmpC-producing Enterobacteriaceae were rarely isolated in LTCF residents in Milan and Piacenza, but $24.3 \%$ of LTCF residents in Bolzano were colonized by $M$. morganii expressing a high-level DHA-AmpC phenotype; $b l a_{\mathrm{DHA}^{-}}$ type genes in LTCF isolates have previously been found in a few E. coli and K. pneumoniae strains from Korea [30], but to our knowledge have not yet been reported in Italian LTCFs.

Carbapenemase-producing enterobacteria were not found in LTCF residents in Piacenza, rarely in Bolzano $(1.6 \%)$ and more frequently in Milan (7.6\%). As found in previous studies of carbapenemase-producing Enterobacteriaceae from Bolzano [22, 23, 31], the VIM-1producing $E$. coli and $C$. amalonaticus isolates from residents in this study were also positive for bla $a_{\mathrm{SHV}-12}$. In the present study, all carbapenemase producers from Milan, except an E. cloacae complex isolate expressing a $b_{l} a_{\mathrm{VIM}-1}$ gene, had KPC-type enzymes; similar results have been reported by other Italian studies in LTCF residents [25, 32, 33]. Carbapenemase-producing enterobacteria, especially KPC-producing $K$. pneumoniae, are epidemically spread in Italy [34] and the emergence of this MDR phenotype in LTCFs is worrying, expanding the reservoir of this health care threat. Nevertheless, as previously summarized [4], carbapenemase-producing Enterobacteriaceae are still rare in Italian LTCF residents; the reasons are probably multifactorial, comprising clinical characteristics of the enrolled residents [35] and the low carbapenem selective pressure in LTCFs. On average, only $1.1 \%$ of residents enrolled in our screening study received carbapenems within the previous 3 months (data not shown). Nevertheless, a carbapenemase-producing enterobacteria prevalence of 7.6\% (mainly KPC-producing K. pneumoniae), reported here for the LTCF in Milan, gives rise to concern and has to be addressed by future hygiene and antibiotic stewardship measures.

This study shows the emergence of carbapenemaseproducing $P$. aeruginosa in LTCF residents in Piacenza, identifying single isolates with $b l a_{\mathrm{VIM}}$-type and $b l a_{\mathrm{GES}-5}$ determinants. $P$. aeruginosa expressing $b l a_{\mathrm{VIM}}$-type determinants is widely spread in Italy [36], and an outbreak of GES-5-producing $P$. aeruginosa was reported from a LTCF in Japan [37]. Moreover, the ES $\beta \mathrm{L}$ genes $b l a_{\mathrm{GES}-1}$ and $b l a_{\mathrm{BEL}}$-like were found in two and one $P$. aeruginosa isolates, respectively; the latter rarely detected $\beta$ lactamase was previously recovered in $P$. aeruginosa strains from Belgium [18]. A. baumannii producing OXA-23 carbapenemases have an epidemic diffusion in Italy [38], reflected in the present study by the isolation of this resistance type from LTCF residents in Milan (1.9\%) and Piacenza (5.8\%).

MRSA colonization prevalence here reported ranged widely in the surveyed LTCFs $(5.7,14.8$ and $24.0 \%$ in Milan, Piacenza and Bolzano, respectively), similar to other Italian studies [25, 39, 40]. Varying MRSA 
Table 3 Resident's risk factors for ESßL and MRSA colonization (cumulative data: Milan, Piacenza, Bolzano)

\begin{tabular}{|c|c|c|c|c|c|c|c|c|c|c|c|c|}
\hline & \multirow[b]{2}{*}{$\begin{array}{l}\mathrm{ESBL}, \% \\
(n=203)\end{array}$} & \multirow[b]{2}{*}{$\begin{array}{l}\text { No ESBL, } \% \\
(n=137)\end{array}$} & \multicolumn{2}{|l|}{$\begin{array}{l}\text { Univariate } \\
\text { analysis }\end{array}$} & \multicolumn{2}{|l|}{$\begin{array}{l}\text { Multivariate } \\
\text { analysis }\end{array}$} & \multirow[b]{2}{*}{$\begin{array}{l}\text { MRSA, \% } \\
(n=45)\end{array}$} & \multirow[b]{2}{*}{$\begin{array}{l}\text { No MRSA, \% } \\
(n=295)\end{array}$} & \multicolumn{2}{|l|}{$\begin{array}{l}\text { Univariate } \\
\text { analysis }\end{array}$} & \multicolumn{2}{|l|}{$\begin{array}{l}\text { Multivariate } \\
\text { analysis }\end{array}$} \\
\hline & & & $\begin{array}{l}\text { OR } \\
\text { (Cl 95\%) }\end{array}$ & $p$ & $\begin{array}{l}\text { OR } \\
\text { (CI 95\%) }\end{array}$ & $p$ & & & $\begin{array}{l}\text { OR } \\
\text { (CI 95\%) }\end{array}$ & $p$ & $\begin{array}{l}\text { OR } \\
\text { (Cl 95\%) }\end{array}$ & $p$ \\
\hline Male sex & 34.9 & 31.3 & $\begin{array}{l}1.17 \\
(0.74-1.86)\end{array}$ & 0.49 & & & 51.1 & 30.8 & $\begin{array}{l}2.34 \\
(1.24-4.42)\end{array}$ & 0.008 & $\begin{array}{l}2.31 \\
(1.16-4.59)\end{array}$ & 0.01 \\
\hline Age $\geq 86$ years & 52.7 & 49.2 & $\begin{array}{l}1.15 \\
(0.74-1.78)\end{array}$ & 0.53 & & & 39.0 & 53.0 & $\begin{array}{l}0.56 \\
(0.29-1.10)\end{array}$ & 0.09 & & \\
\hline $\begin{array}{l}\text { Antibiotics in preceding } \\
3 \text { months }\end{array}$ & 39.9 & 23.3 & $\begin{array}{l}2.17 \\
(1.34-3.54)\end{array}$ & 0.001 & $\begin{array}{l}1.74 \\
(1.02-2.98)\end{array}$ & 0.04 & 37.7 & 32.5 & $\begin{array}{l}1.25 \\
(0.65-2.41)\end{array}$ & 0.48 & & \\
\hline Fluoroquinolones & 7.8 & 5.8 & $\begin{array}{l}1.38 \\
(0.57-3.32)\end{array}$ & 0.47 & & & 15.5 & 5.7 & $\begin{array}{l}3.01 \\
(1.17-7.73)\end{array}$ & 0.02 & $\begin{array}{l}3.59 \\
(1.26-10.25)\end{array}$ & 0.01 \\
\hline Penicillins & 7.3 & 2.9 & $\begin{array}{l}2.65 \\
(0.86-8.17)\end{array}$ & 0.09 & & & 11.1 & 4.7 & $\begin{array}{l}2.50 \\
(0.85-7.34)\end{array}$ & 0.09 & & \\
\hline Cephalosporins & 14.2 & 6.5 & $\begin{array}{l}2.37 \\
(1.08-5.18)\end{array}$ & 0.03 & & & 4.4 & 12.2 & $\begin{array}{l}0.33 \\
(0.07-1.44)\end{array}$ & 0.14 & & \\
\hline Dementia & 63.0 & 66.4 & $\begin{array}{l}0.86(0.54- \\
1.36)\end{array}$ & 0.52 & & & 62.2 & 64.7 & $\begin{array}{l}0.89 \\
(0.47-1.71)\end{array}$ & 0.74 & & \\
\hline $\begin{array}{l}\text { Peripheral vascular } \\
\text { disease }\end{array}$ & 62.5 & 59.1 & $\begin{array}{l}1.15 \\
(0.74-1.80)\end{array}$ & 0.52 & & & 62.2 & 61.0 & $\begin{array}{l}1.05 \\
(0.55-2.00)\end{array}$ & 0.87 & & \\
\hline Urinary incontinence & 83.2 & 78.8 & $\begin{array}{l}1.33 \\
(0.77-2.31)\end{array}$ & 0.30 & & & 82.2 & 81.3 & $\begin{array}{l}1.06 \\
(0.46-2.40)\end{array}$ & 0.89 & & \\
\hline Diabetes & 18.7 & 18.9 & $\begin{array}{l}0.98 \\
(0.56-1,71)\end{array}$ & 0.30 & & & 26.6 & 17.6 & $\begin{array}{l}1.69 \\
(0.82-3.51)\end{array}$ & 0.15 & & \\
\hline Cancer & 11.8 & 4.3 & $\begin{array}{l}2.92 \\
(1.16-7.36)\end{array}$ & 0.02 & $\begin{array}{l}3.47 \\
(1.32-9.16)\end{array}$ & 0.01 & 4.4 & 9.5 & $\begin{array}{l}0.44 \\
(0.10-1.93)\end{array}$ & 0.27 & & \\
\hline Decubitus ulcer & 9.8 & 5.1 & $\begin{array}{l}2.03 \\
(0.83-4.94)\end{array}$ & 0.12 & & & 6.6 & 8.1 & $\begin{array}{l}0.80 \\
(0.23-2.79)\end{array}$ & 0.73 & & \\
\hline $\begin{array}{l}\text { Chronic obstructive } \\
\text { pulmonary disease }\end{array}$ & 11.8 & 14.6 & $\begin{array}{l}0.78 \\
(0.41-1.48)\end{array}$ & 0.45 & & & 15.5 & 12.5 & $\begin{array}{l}1.28 \\
(0.53-3.08)\end{array}$ & 0.57 & & \\
\hline $\begin{array}{l}\text { Physical disability } \\
\text { (Barthel immobility } \\
\text { score of } 0 \text { ) }\end{array}$ & 47.7 & 32.3 & $\begin{array}{l}1.91 \\
(1.21-3.02)\end{array}$ & 0.005 & $\begin{array}{l}2.10 \\
(1.15-3.83)\end{array}$ & 0.01 & 37.7 & 41.0 & $\begin{array}{l}0.87 \\
(0.45-1.66)\end{array}$ & 0.67 & & \\
\hline Coma & 6.9 & 4.3 & $\begin{array}{l}1.61 \\
(0.60-4.31)\end{array}$ & 0.33 & & & 6.6 & 5.7 & $\begin{array}{l}1.16 \\
(0.32-4.15)\end{array}$ & 0.81 & & \\
\hline Any medical device & 32.5 & 13.1 & $\begin{array}{l}3.18 \\
(1.79-5.66)\end{array}$ & $\begin{array}{l}< \\
0.001\end{array}$ & $\begin{array}{l}2.81 \\
(1.44-5.47)\end{array}$ & 0.002 & 33.3 & 23.3 & $\begin{array}{l}1.63 \\
(0.83-3.21)\end{array}$ & 0.15 & & \\
\hline $\begin{array}{l}\text { Percutaneous enteral } \\
\text { gastrostomy tube }\end{array}$ & 15.7 & 6.5 & $\begin{array}{l}2.66 \\
(1.22-5.77)\end{array}$ & 0.01 & & & 11.1 & 12.2 & $\begin{array}{l}0.89 \\
(0.33-2.42)\end{array}$ & 0.83 & & \\
\hline Tracheostomy tube & 4.9 & 2.1 & $\begin{array}{l}2.31 \\
(0.62-8.56)\end{array}$ & 0.21 & & & 4.4 & 3.7 & $\begin{array}{l}1.20 \\
(0.25-5.60)\end{array}$ & 0.81 & & \\
\hline Urinary catheter & 15.7 & 4.3 & $\begin{array}{l}4.08 \\
(1.66-10.06)\end{array}$ & 0.002 & & & 20.0 & 9.8 & $\begin{array}{l}2.29 \\
(1.00-5.23)\end{array}$ & 0.04 & $\begin{array}{l}2.61 \\
(0.06-6.43)\end{array}$ & 0.03 \\
\hline Nasogastric tube & 5.9 & 0.7 & $\begin{array}{l}8.54 \\
(1.09-66.49)\end{array}$ & 0.04 & & & 4.4 & 3.7 & $\begin{array}{l}1.20 \\
(0.25-5.60)\end{array}$ & 0.81 & & \\
\hline $\begin{array}{l}\text { Length of stay in } \\
\text { LTCF }<6 \text { months }\end{array}$ & 15.6 & 11.9 & $\begin{array}{l}1.36 \\
(0.71-2.61)\end{array}$ & 0.34 & & & 16.6 & 13.7 & $\begin{array}{l}1.25 \\
(0.51-3.00)\end{array}$ & 0.61 & & \\
\hline $\begin{array}{l}\text { Hospital admission in } \\
\text { previous } 12 \text { months }\end{array}$ & 24.2 & 27.0 & $\begin{array}{l}0.87 \\
(0.53-1.43)\end{array}$ & 0.58 & & & 37.7 & 23.4 & $\begin{array}{l}1.97 \\
(1.02-3.81)\end{array}$ & 0.04 & & \\
\hline Infection & 5.4 & 2.9 & $\begin{array}{l}1.90 \\
(0.59-6.11)\end{array}$ & 0.27 & & & 8.8 & 3.7 & $\begin{array}{l}2.51 \\
(0.76-8.28)\end{array}$ & 0.12 & & \\
\hline
\end{tabular}

ND: not determined; factors included in multivariate analysis are in italics. For multivariate analysis only significant values are shown 
colonization prevalence, ranging from close to zero up to levels higher than 37\%, has been reported in European studies [4].

Colonization by VRE in the present study was highly variable, ranging from 0.8 to $20.2 \%$. VRE-carriage in European LTCF residents was found to be low, ranging from $0.0-3 \%[28,41,42]$.

For Enterobacteriaceae significant differences in colonization frequencies of LTCF residents were found: i) for CTX-M-type ES $\beta$ L-producing E. coli between Piacenza (highest prevalence) and Bolzano, ii) for high-level AmpCproducing M. morganii (highest prevalence in Bolzano), iii) for carbapenemase producers, with highest prevalence in Milan, iv) for carbapenemase-producing A. baumannii, showing highest prevalence in Piacenza, and v) for MRSA and VRE, most prevalent in Milan. Therefore, no clear picture of general colonization differences can be deduced from overall colonization prevalence data.

A variety of risk factors for MRSA and ES $\beta L$ colonization have previously been reported [4]; many of these have also been analyzed in the present survey. Interestingly, male residents carried a more than double risk for MRSA carriage when compared with female residents, probably because of the higher frequencies of other risk factors in males (administration of any antibiotic within the previous 3 months, hospitalization within the previous 12 months and coma), predisposing men rather than women to MRSA acquisition. Moreover, in our study the trend for an inverse correlation ( $p$ $=0.09$ ) between age $>85$ years and MRSA prevalence was associated with a significantly lower percentage of male residents $>85$ years, compared to females; similar results have been found by other authors [43]. In the present survey, administration of cephalosporins during the previous 3 months resulted to be an independent risk factor for ES $\beta$ L colonization; the LTCFs in Piacenza registered the highest consumption of cephalosporins, correlating with highest ES $\beta$ L prevalence in LTCF residents from Piacenza. Other independent risk factors for ES $\beta \mathrm{L}$ colonization were physical disability, the presence of any invasive medical device and cancer. Whereas no significant differences were found between residents in the three Provinces for cancer as risk factor, physical disability and the presence of any medical device showed highest prevalence in the LTCF in Bolzano; nonetheless, LTCF residents in Bolzano had the lowest ES $\beta$ L prevalence in the present screening study.

Therefore, further factors may have contributed to the observed differences, comprising staff/resident ratio and practiced hygiene and infection control measures [44]. The LTCF in Bolzano showed the highest staff/resident ratio, and understaffing has been shown to be a risk factor for colonization of LTCF residents by MDR organisms [2]. All of the surveyed LTCFs in the present study follow hygiene, infection prevention and control measures according to guidelines of The Society for Healthcare Epidemiology of America (SHEA) and The Association for Professionals in Infection Control and Epidemiology (APIC) [45]. Nonetheless, the Bolzano LTCF had introduced enforced hygiene measures, according to the World Health Organization guidelines [46], after the 2008 screening study, showing an ES $\beta$ L colonization prevalence of $64.0 \%$ in LTCF residents [22]; colonization frequency decreased significantly to $49.0 \%(p=0.02)$ in 2012 [23], arriving at a slightly higher percentage of $53.0 \%$ in 2016 , but other factors such as changed case mixes and risk factors may also have contributed to this decrease in ES $\beta \mathrm{L}$ prevalence [23].

Significant differences in antibiotic resistance epidemiology of blood culture isolates, used as a proxy for the general local antibiotic resistance epidemiology, were registered, as derived from European Antimicrobial Resistance Surveillance Network (EARS-Net) data for 2016 [26]. Specifically, we found the following antibiotic resistance data referred to the geographic regions of Milan, Piacenza and Bolzano, respectively: $E$. coli third generation cephalosporin-resistant: $22.1 \%$ (29/131), 29.4\% (71/259) and 17.8\% (56/314); K. pneumoniae carbapenem-resistant: 29.2\% (7/24), 13.5\% (10/74) and $6.2 \%$ (4/64); A. baumannii carbapenem-resistant: $50.0 \%$ $(1 / 2) 100.0 \%,(24 / 24)$ and $0.0 \%(0 / 2)$; MRSA: $36.0 \%$ (18/50), 49.7\% (82/165) and 14.6\% (20/137); E. faecalis VRE: 0.0\% (0/ 20), $2.4 \%(2 / 83)$ and $0.0 \%$ (0/41); E. faecium VRE: $10.0 \%$ (1/ 10), $22.2 \%(6 / 27)$ and $8.0 \%(2 / 25)$. This data for blood culture isolates, compared with our LTCF screening data, correlates well for ES $\beta$ L-producing E. coli, carbapenemresistant $K$. pneumoniae and $A$. baumannii; on the other hand, no correlation for MRSA and VRE can be derived. Patient transfer between acute-care facilities and LTCFs contribute to the diffusion of MDR organisms in both settings; such bi-directional movement of MDR bacteria, related to acute systemic infections, might be more significant for Enterobacteriaceae and A. baumannii than for MRSA and VRE.

Moreover, the snapshot approach used in this study might lead to the sudden increase in prevalence of a specific resistance phenotype, as shown for high-level AmpC-producing M. morganii detected in 2016 from Bolzano LTCF residents [5], which could be a transient phenomenon. Similarly, the high prevalence of VRE in LTCF residents from Milan could be due to a transitory local epidemic event.

Finally, the local circulation of highly transmissible clones, for example ESßL-producing E. coli, KPCproducing $K$. pneumoniae and OXA-23-producing $A$. baumannii could contribute to the explanation of the here reported screening results [38, 47].

This study has some limitations. First, it has been done in only four LTCFs, located in three different Provinces 
in Northern Italy, and therefore data may not be extrapolated to other Italian LTCFs with differing characteristics. Second, the number of LTCF residents participating in the study was variable, ranging from $34 \%$ in Milan up to $100 \%$ in Bolzano. Third, we did not use an enrichment step during the laboratory analysis; this limitation is partially compensated by using $4-5$ different specimen types for the screening of MDR bacteria. Fourth, different sample types, types of media and laboratory methodologies have been used in the three laboratories processing the samples from the different LTCFs. Fifth, molecular characterization and typing of isolates in the 2016 study was limited, not including pulsed-field gel electrophoresis (PFGE) and sequence typing (ST) of isolates and therefore not permitting the identification of epidemic clusters. Finally, screening of healthcare workers has been done only in one of the enrolled LTCFs [5], but not in the other surveyed facilities. Despite these limitations, the strength of our study is the comparison of colonization prevalence between LTCFs located in three different Provinces, comparing it also with differences in risk factors for colonization and in the local epidemiology of invasive isolates.

\section{Conclusions}

We performed a multicenter point-prevalence study in LTCFs located in three different Provinces in Northern Italy and found high colonization prevalence of LTCF residents for MDR organisms, especially ES $\beta$ L-producing $E$. coli. Variability between the different facilities was noticeable also for other MDR organisms. Differences can be partially explained by i) differences in risk factors for colonization by MDR organisms, ii) changes in resident populations and staff/resident ratios, iii) applied hygiene measures and iv) differences in the local epidemiology of antibiotic resistance of clinical isolates. This widespread diffusion of MDR bacteria in LTCFs of three Italian Provinces confirms that these healthcare facilities are an important reservoir for MDR organisms. Future efforts should focus on screening activities, infection control strategies tailored on the complex aspects of LTCFs and implementation of antibiotic stewardship programs.

\section{Additional file}

Additional file 1: Table S1. Oligonucleotides used for PCR and sequencing. (DOCX $17 \mathrm{~kb}$ )

\footnotetext{
Abbreviations

AMCLI: Association of Italian Clinical Microbiologist; APIC: The Association for Professionals in Infection Control and Epidemiology; CTX-M: CefotaximaseMunich type Extended-Spectrum $\beta$-Lactamase; EARS-Net: European Antimicrobial Resistance Surveillance Network; ESßL: Extended-Spectrum $\beta$ Lactamase; EUCAST: European Committee on Antimicrobial Susceptibility Testing; GLISTer: Gruppo di Lavoro per lo Studio delle Infezioni nelle Residenze Sanitarie Assistite e Strutture Assimilabili; KPC: Klebsiella
}

Pneumoniae Carbapenemase; LTCF: Long-Term Care Facility; MBL: Metallo- $\beta$ Lactamase; MDR: Multidrug-Resistant; MRSA: Methicillin-Resistant Staphylococcus aureus; PCR: Polymerase Chain Reaction; PFGE: Pulsed-Field Gel Electrophoresis; SHEA: The Society for Healthcare Epidemiology of America; ST: Sequence Typing; VIM: Verona Integron-Encoded Metallo- $\beta$ Lactamase; VRE: Vancomycin-Resistant Enterococci

\section{Acknowledgements}

We wish to thank the residents and their relatives who agreed to participate in this study, the managements and staff of the long-term care facilities and the microbiology laboratories for excellent advice and technical assistance. We are grateful to Dr. Francesco Luzzaro, Lecco, for providing regional EARS-Net antimicrobial resistance data from the geographic region of Milan.

Members of the GLISTer group are kindly acknowledged: Laura Pagani, Massimo Confalonieri, Richard Aschbacher, Claudio Farina, Paolo Fazii, Francesco Luzzaro, Pier Giorgio Montanera.

Prof. Roberta Migliavacca is a member of the European Society of Clinical Microbiology and Infectious Diseases - Study Group for Infections in the Elderly (ESGIE) and would like to thank ESGIE members for their stimulating support to write this manuscript.

\section{Funding}

The microbiological work was partially funded by a research grant of the "Fondo Ricerca e Giovani 2016" -University of Pavia and by an unconditional grant of Cepheid.

\section{Availability of data and materials}

The datasets generated during and/or analysed during the current study are available from the corresponding author on reasonable request.

\section{Authors' contributions}

$E N,{ }^{1} M C, V M M, R M$ performed molecular analysis; RDA, ${ }^{3} M C, E P, A M, F S, G S$ provided patient's sample data; EF, CR, RA performed and interpreted phenotypic investigations; RA, RM, LP analyzed and interpreted results; RA was a major contributor in writing the manuscript. All authors read and approved the final manuscript.

\section{Ethics approval and consent to participate}

The study was approved by the Ethics Committees of the three referring hospitals.

\section{Consent for publication}

Not applicable.

\section{Competing interests}

The authors declare that they have no competing interests.

\section{Publisher's Note}

Springer Nature remains neutral with regard to jurisdictional claims in published maps and institutional affiliations.

\section{Author details}

'Department of Clinical Surgical Diagnostic and Pediatric Sciences, Laboratory of Microbiology and Clinical Microbiology, University of Pavia, Via Brambilla 74, 27100 Pavia, Italy. ${ }^{2}$ Laboratory of Clinical Microbiology, ASP "Golgi-Redaelli", via Bartolomeo d'Alviano 78, 20146 Milan, Italy. ${ }^{3}$ O.U. of Microbiology, Azienda Sanitaria Locale di Piacenza, Piacenza, Italy. ${ }^{4}$ Geriatric Unit, Comprensorio Sanitario di Bolzano, Bolzano, Italy. ${ }^{5}$ Microbiology and Virology Laboratory, Comprensorio Sanitario di Bolzano, Bolzano, Italy.

Received: 19 December 2017 Accepted: 20 February 2018 Published online: 06 March 2018

\section{References}

1. Istituto nazionale di statistica. http://www.istat.it/. Accessed 24 July 2017.

2. Moro ML, Gagliotti C. Antimicrobial resistance and stewardship in long-term care settings. Future Med. 2013;8:1011-25.

3. Cassone M, Mody L. Colonization with multi-drug resistant organisms in nursing homes: scope, importance, and management. Curr Geriatr Rep. 2015;4:87-95. 
4. Aschbacher R, Pagani E, Confalonieri M, Farina C, Fazii P, Luzzaro F, et al. Review on colonization of residents and staff in Italian long-term care facilities by multidrug-resistant bacteria compared with other European countries. Antimicrob Resist Infect Control. 2016:5:33.

5. March A, Aschbacher R, Sleghel F, Soelva S, Kaczor M, Migliavacca R, et al. Colonization of residents and staff of an Italian long-term care facility and an adjacent acute-care hospital geriatric unit by multidrug-resistant bacteria. New Microbiol. 2017:40:258-63.

6. The European Committee on Antimicrobial Susceptibility Testing. Breakpoint tables for interpretation of MICs and zone diameters. Version 6.0, 2016. http://www.eucast.org

7. Pagani L, Dell'Amico E, Migliavacca R, D'Andrea MM, Giacobone E, Amicosante G, et al. Multiple CTX-M-type extended-spectrum-lactamases in nosocomial isolates of Enterobacteriaceae from a hospital in Northern Italy. J Clin Microbiol. 2003:41:4264-9.

8. Eckert C, Gautier V, Arlet G. DNA sequence analysis of the genetic environment of various blaCTX-M genes. J Antimicrob Chemother 2006; 57:14-23.

9. Perilli M, Dell'Amico E, Segatore B, De Massis RR, Bianchi C, Luzzaro F, et al. Molecular characterization of extended-spectrum $\beta$-lactamases produced by nosocomial isolates of Enterobacteriaceae from an Italian nationwide survey. J Clin Microbiol. 2002:40:611-4.

10. Rasheed JK, Jay C, Metchock B, Berkowitz F, Weigel L, Crellin J, et al. Evolution of extended-spectrum beta-lactam resistance (SHV-8) in a strain of Escherichia coli during multiple episodes of bacteremia. Antimicrob Agents Chemother. 1997:41:647-53.

11. Yigit H, Queenan AM, Anderson GJ, Domenech-Sanchez A, Biddle JW, Steward CD, et al. Novel carbapenem-hydrolyzing-lactamase, KPC-1, from a carbapenem-resistant strain of Klebsiella pneumoniae. Antimicrob Agents Chemother. 2001;45:1151-61.

12. Poirel $L$, Walsh TR, Cuvillier V, Nordmann P. Multiplex PCR for detection of acquired carbapenemase genes. Diagn Microbiol Infect Dis. 2011;70:119-23.

13. Migliavacca R, Docquier JD, Mugnaioli C, Amicosante G, Daturi R, Lee K. Simple microdilution test for detection of metallo-beta-lactamase production in Pseudomonas aeruginosa. J Clin Microbiol. 2002:40:4388-90.

14. Lagatolla C, Tonin EA, Monti-Bragadin C, Dolzani L, Gombac F, Bearzi C, et al. Endemic carbapenem-resistant Pseudomonas aeruginosa with acquired metallo- $\beta$-lactamase determinants in European hospital. Emerg Infect Dis. 2004;10:535-8.

15. Poirel L, Le Thomas I, Naas T, Karim A, Nordmann P. Biochemical sequence analyses of GES-1, a novel class a extended-spectrum $\beta$-lactamase, and the class 1 integron In52 from Klebsiella pneumoniae. Antimicrob Agents Chemother. 2000:44:622-32.

16. Woodford N, Ellington MJ, Coelho JM, Turton JF, Ward ME, Brown S, et al. Multiplex PCR for genes encoding prevalent OXA carbapenemases in Acinetobacter spp. Int J Antimicrob Agents. 2006;27:351-3.

17. Poirel L, Docquier J-D, De Luca F, Verlinde A, Ide L, Rossolini GM, et al. BEL2 , an extended-Spectrum $\beta$-lactamase with increased activity toward expanded-Spectrum Cephalosporins in Pseudomonas aeruginosa. Antimicrob Agents Chemother. 2010;54:533-5.

18. Higgins $P G$, Lehmann M, Seifert H. Inclusion of OXA-143 primers in a multiplex polymerase chain reaction (PCR) for genes encoding prevalent OXA carbapenemases in Acinetobacter spp. Int J Antimicrob Agents. 2010;35:305.

19. Turton JF, Ward ME, Woodford N, Kaufmann ME, Pike R, Livermore DM, et al. The role of ISAba1 in expression of OXA carbapenemase genes in Acinetobacter baumannii. FEMS Microbiol Lett. 2006;258:72-7.

20. D'Andrea MM, Nucleo E, Luzzaro F, Giani T, Migliavacca R, Vailati F. CMY-16, a novel acquired AmpC-type $\beta$-lactamase of the CMY/LAT lineage in multifocal monophyletic isolates of Proteus mirabilis from northern Italy. Antimicrob Agents Chemother. 2006:50:618-24.

21. National Center for Biotechnology Information (NCBI). Basic Local Alignment Search Tool (BLAST). http://blast.ncbi.nlm.nih.gov/Blast.cgi. Accessed 24 July 2017.

22. March A, Aschbacher R, Dhanji H, Livermore DM, Böttcher A, Sleghel $F$, et al. Colonization of residents and staff of a long-term-care facility and adjacent acute-care hospital geriatric unit by multiresistant bacteria. Clin Microbiol Infect. 2010;16:934-44.

23. March A, Aschbacher R, Pagani E, Sleghel F, Soelva G, Hopkins KL, et al. Changes in colonization of residents and staff of a long-term care facility and an adjacent acute-care hospital geriatric unit by multidrug-resistant bacteria over a four-year period. Scand J Infect Dis. 2014;46:114-22.

24. Arnoldo L, Migliavacca R, Regattin L, Raglio A, Pagani L, Nucleo E, et al. Prevalence of urinary colonization by extended spectrum-beta-lactamase
Enterobacteriaceae among catheterised inpatients in Italian long-term care facilities. BMC Infect Dis. 2013;13:124.

25. Giufrè M, Ricchizzi E, Accogli M, Barbanti F, Monaco M, Pimentel de Araujo F, et al. Colonization by multidrug-resistant organisms in long-term care facilities in Italy: a point-prevalence study. Clin Microbiol Infect. 2017;23:961-7.

26. European Centre for Disease Prevention and Control. https://ecdc.europa. eu/. Accessed 24 July 2017

27. Rooney PJ, O'Leary MC, Loughrey AC, McCalmont M, Smyth B, Donaghy P, et al. Nursing homes as a reservoir of extended-spectrum beta-lactamase (ESBL)-producing ciprofloxacin-resistant Escherichia coli. J Antimicrob Chemother. 2009;64:635-41.

28. Ludden C, Cormican M, Vellinga A, Johnson JR, Austin B, Morris D. Colonisation with ESBL-producing and carbapenemase-producing Enterobacteriaceae, vancomycin-resistant enterococci, and meticillin-resistant Staphylococcus aureus in a long-term care facility over one year. BMC Infect Dis. 2015;15:168

29. Rodrigues C, Mendes AC, Sima F, Bavlovič J, Machado E, Novais Â, et al. Longterm care facility (LTCF) residents colonized with multidrug-resistant (MDR) Klebsiella pneumoniae lineages frequently causing infections in Portuguese clinical institutions. Infect Control Hosp Epidemiol. 2017;38:1127-30.

30. Yoo JS, Byeon J, Yang J, Yoo Jl, Chung GT, Lee YS. High prevalence of extended-spectrum beta-lactamases and plasmid-mediated AmpC betalactamases in Enterobacteriaceae isolated from long-term care facilities in Korea. Diagn Microbiol Infect Dis. 2010;67:261-5.

31. Carattoli A, Aschbacher R, March A, Larcher C, Livermore DM, Woodford N. Complete nucleotide sequence of the IncN plasmid pKOX105 encoding VIM-1, QnrS1 and SHV-12 proteins in Enterobacteriaceae from Bolzano, Italy compared with IncN plasmids encoding KPC enzymes in the USA. J Antimicrob Chemother. 2010;65:2070-5.

32. Del Franco M, Paone L, Novati R, Giacomazzi CG, Bagattini M, Galotto C, et al, Molecular epidemiology of carbapenem resistant Enterobacteriaceae in Valle d'Aosta region, Italy, shows the emergence of KPC-2 producing Klebsiella pneumoniae clonal complex 101 (ST101 and ST1789). BMC Microbiol. 2015;15:260

33. Piazza A, Caltagirone M, Bitar I, Nucleo E, Spalla M, Fogato E, et al. Emergence of Escherichia coli sequence type 131 (ST131) and ST3948 with KPC-2, KPC-3 and KPC-8 carbapenemases from a long-term care and rehabilitation facility (LTCRF) in northern Italy. Adv Exp Med Biol. 2016;901:77-89.

34. Giani T, Pini B, Arena F, Conte V, Bracco S, Migliavacca R, et al. Epidemic diffusion of KPC carbapenemase-producing Klebsiella pneumoniae in Italy: results of the first countrywide survey, 15 may to 30 June 2011. Euro Surveill. 2013;18(22)

35. Prasad N, Labaze G, Kopacz J, Chwa S, Platis D, Pan CX, et al. Asymptomatic rectal colonization with carbapenem-resistant Enterobacteriaceae and Clostridium difficile among residents of a long-term care facility in New York City. Am J Infect Control. 2016:44:525-32.

36. Rossolini GM, Luzzaro F, Migliavacca R, Mugnaioli C, Pini B, De Luca F, et al. First countrywide survey of acquired metallo-beta-lactamases in gramnegative pathogens in Italy. Antimicrob Agents Chemother. 2008;52:4023-9.

37. Kanayama A, Kawahara R, Yamagishi T, Goto K, Kobaru Y, Takano M, Morisada K, et al. Successful control of an outbreak of GES-5 extendedspectrum $\beta$-lactamase-producing Pseudomonas aeruginosa in a long-term care facility in Japan. J Hosp Infect. 2016;93:35-41.

38. Principe L, Piazza A, Giani T, Bracco S, Caltagirone MS, Arena F, et al. Epidemic diffusion of OXA-23-producing Acinetobacter baumannii isolates in Italy: results of the first cross-sectional countrywide survey. J Clin Microbiol. 2014;52:3004-10.

39. Brugnaro P, Fedeli U, Pellizzer G, Buonfrate D, Rassu M, Boldrin C, et al. Clustering and risk factors of methicillin-resistant Staphylococcus aureus carriage in two Italian long-term care facilities. Infection. 2009;37:216-21.

40. Monaco M, Bombana E, Trezzi L, Regattin L, Brusaferro S, Pantosti A, et al. Methicillin-resistant Staphylococcus aureus colonizing residents and staff members in a nursing home in northern Italy. J Hosp Infect. 2009;73:182-4.

41. Jans B, Schoevaerdts D, Huang TD, Berhin C, Latour K, Bogaerts P, et al. Epidemiology of multidrug-resistant microorganisms among nursing home residents in Belgium. PLoS One. 2013;8:e64908.

42. Hogardt M, Proba P, Mischler D, Cuny C, Kempf VA, Heudorf U. Current prevalence of multidrug-resistant organisms in long-term care facilities in the Rhine-main district, Germany, 2013. Euro Surveill. 2015;20(26)

43. Nillius D, von Müller L, Wagenpfeil S, Klein R, Herrmann M. Methicillinresistant Staphylococcus aureus in Saarland, Germany: the long-term care facility study. PLoS One. 2016; https://doi.org/10.1371/journal.pone.0153030. 
44. Dyar OJ, Pagani L, Pulcini C. Strategies and challenges of antimicrobial stewardship in long-term care facilities. Clin Microbiol Infect. 2015;21:10-9.

45. Smith PW, Bennett G, Bradley S, Drinka P, Lautenbach E, Marx J, et al. SHEA/ APIC guideline: infection prevention and control in the long-term care facility. Infect Control Hosp Epidemiol. 2008;29:785-814.

46. http://apps.who.int/iris/bitstream/10665/44102/1/9789241597906_eng.pdf. Accessed 24 July 2017.

47. Mathers AJ, Peirano G, Pitout JD. The role of epidemic resistance plasmids and international high-risk clones in the spread of multidrug-resistant Enterobacteriaceae. Clin Microbiol Rev. 2015;28:565-91.

Submit your next manuscript to BioMed Central and we will help you at every step:

- We accept pre-submission inquiries

- Our selector tool helps you to find the most relevant journal

- We provide round the clock customer support

- Convenient online submission

- Thorough peer review

- Inclusion in PubMed and all major indexing services

- Maximum visibility for your research

Submit your manuscript at www.biomedcentral.com/submit
Biomed Central 